\title{
Modern Brachytherapy Techniques - Real-Time High Dose Rate Brachytherapy for Prostate Cancer
}

\author{
D. MiszCZAK \\ Maria Skłodowska-Curie Memorial Institute, Centre of Oncology, Kraków Division \\ Garncarska 11, 31-115 Kraków, Poland
}

\begin{abstract}
The "real time" technique of high dose rate brachytherapy of the prostate gland is described from the point of view of the medical physicist, as an illustration of modern radiotherapy techniques currently applied at the Centre of Oncology in Krakow. This technique, based on ultrasound imaging and on-line calculations of dose distribution around catheters inserted into the prostate volume in which a radioactive Ir-192 source is moved with the aid of an afterloading apparatus, is performed in operating-room conditions in close cooperation with the physician within stringent time limits.
\end{abstract}

PACS numbers: 87.53.Jw, 87.55.de, 87.56.- -v, 87.63.-d

\section{Introduction}

The general aim of radiotherapy is to deliver the clinically prescribed dose to the tumour (target) volume while sparing surrounding healthy tissues and organs at risk [1]. Several radiotherapy techniques are currently in use, distinguished by means of delivering the dose to the tumour. The patient may be irradiated by external beams of high-energy photons or electrons (teletherapy, see accompanying article [2]), by encapsulated radioactive sources of small dimensions placed in close vicinity of the tumour volume (brachytherapy), or by radioactive pharmaceuticals introduced into the patient's body to be preferentially absorbed in the tumour volume (systemic radiotherapy). Brachytherapy (sometimes referred to as curietherapy or endocurietherapy) was the earliest technique of cancer radiotherapy, where radium needles (many of them prepared by Maria Skłodowska-Curie herself) were placed close to the tumour delivering gammaradiation at relatively low dose rates. Radium-226 needles have now been replaced by sources of higher specific activity and lower photon energy (cf. Table), able to deliver much higher dose rates (high dose rate (HDR) brachytherapy) despite their small physical dimensions, of some $\mathrm{mm}^{3}$. To reduce exposure to the medical personnel operating such sources, afterloading equipment has been developed, whereby the source (or sources) can be remotely introduced into suitable applicators placed inside the patient after completion of preparatory procedures, including computerized planning of the required dose distribution, performed by the medical physicist. A more recent development is the ability to move the source inside the applicator, enabling the medical physicist to further optimise this dose distribution on the basis of $3 \mathrm{D}$ (three-dimensional) reconstruction of the source positions from X-ray (or ultrasound) images of patient anatomy and applicator positions.
TABLE

Selected characteristics of isotopes used in brachytherapy.

\begin{tabular}{c|c|c}
\hline \hline Isotope & $\begin{array}{c}\text { Average }^{a} \text { photon } \\
\text { energy }[\mathrm{MeV}]\end{array}$ & Half-life \\
\hline Ra-226 & $0.24-2.4$ & 1600 years \\
Co-60 & 1.25 & 5.26 years \\
Cs-137 & 0.66 & 30 years \\
Au-198 & 0.41 & 2.7 days \\
Ir-192 & 0.38 & 73.8 days \\
I-125 & 0.028 & 60 days \\
Pd-103 & 0.021 & 17 days \\
\hline
\end{tabular}

${ }^{a}$ These are only approximate values, depending on the type of source encapsulation.

The main advantage of brachytherapy over external beam treatment is its ability to deliver a high dose of radiation within a well-defined volume, with rapid dose rate fall-off outside this volume, owing to the typical $1 / r^{2}$ range - dose rate dependence around a small radioactive source. In radiotherapy of prostate cancer where the target organ lies very close to critical normal tissues (anterior rectal wall and bladder base), the modern approach is to use either external radiotherapy, especially intensity-modulated radiation therapy (IMRT) (see [2]) or real-time brachytherapy where transrectal ultrasound-guided insertion of applicators in conjunction with HDR afterloading enables brachytherapy to be delivered accurately and safely to the prostate gland $[1,3]$. The aim of this work is to briefly describe the physical aspects of the last procedure which is now routinely performed at our Centre. 


\section{Description of the real-time HDR brachytherapy procedure}

The real-time HDR brachytherapy procedure is carried out under sterile operating-theatre conditions inside a HDR bunker, to comply with radiation safety regulations. Prior to his treatment the patient is anaesthetised (spinally or generally) and remains under the care of an anaesthesiologist. Due to anaesthesia the time available for this procedure is limited, so efficient cooperation between the physician-radiotherapist and the medical physicist is essential.

First, a catheter is inserted in the urethra and an ultrasound probe placed in the patient's rectum. The initial virtual plan is prepared by the medical physicist, basing on the ultrasound image of the pelvic area acquired in continuous mode over $1 \mathrm{~mm}$ intervals. The target volume is delineated on each scan by the physician. The calculation of the "virtual" planned dose distribution is performed by the medical physicist on the basis of the assumed number and assumed positions of needles into which the Ir-192 source will later be moved, and software-programmable dwell positions and dwell times of the source inside these needles. Since clinical complications of prostate cancer radiotherapy may result from high doses delivered to the portions of the urethra and rectum in close proximity to the sources, an optimum plan is one that shows a homogeneous dose distribution within the target volume and, if possible, a "cold spot" (low dose area) around the urethra. This is achieved by optimising, via computer dose planning system calculations, the dwell positions and dwell times along the length of each applicator at their assumed geometric positions. Once optimised and accepted, this virtual plan guides needle insertion around the target volume, performed by the physician (Fig. 1). A special template is used to aid the physician in accurately positioning the required number of needles around the prostate region.

After all needles have been inserted, ultrasound image acquisition is repeated. Usually, the shape of the prostate and its volume change following needle insertion, so the prostate, rectum and urethra need to be contoured for the second time by the physician. The position of each needle is then modified within the treatment planning system to update their coordinates. Sometimes the actual position of a given needle may differ with respect to its original (virtual) value by several millimeters. Next, optimisation of the dose distribution must be performed for updated needle positions, which usually entails changing the dwell times of each source position in the needles. This updated plan is called the live plan (Fig. 2) and, if found to be clinically acceptable, is applied in the treatment procedure. For a treatment plan to be clinically acceptable $90 \%$ of the therapeutic dose should be delivered to $95 \%$ of the volume of the prostate gland and no more than $10 \%$ of the volume of the urethra should receive a dose higher than $125 \%$ of the planned dose [4]. After the live plan is accepted by the physician, it is transferred via the local network and used to guide the operation of the

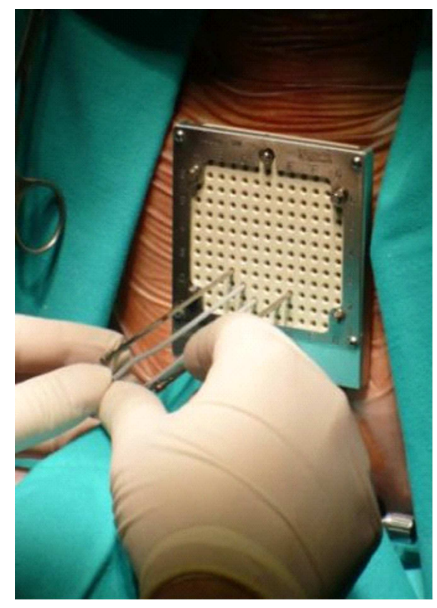

Fig. 1. Insertion of needles around the target volume, performed by the physician. Let us note the template used to aid in accurately positioning the required number of needles.

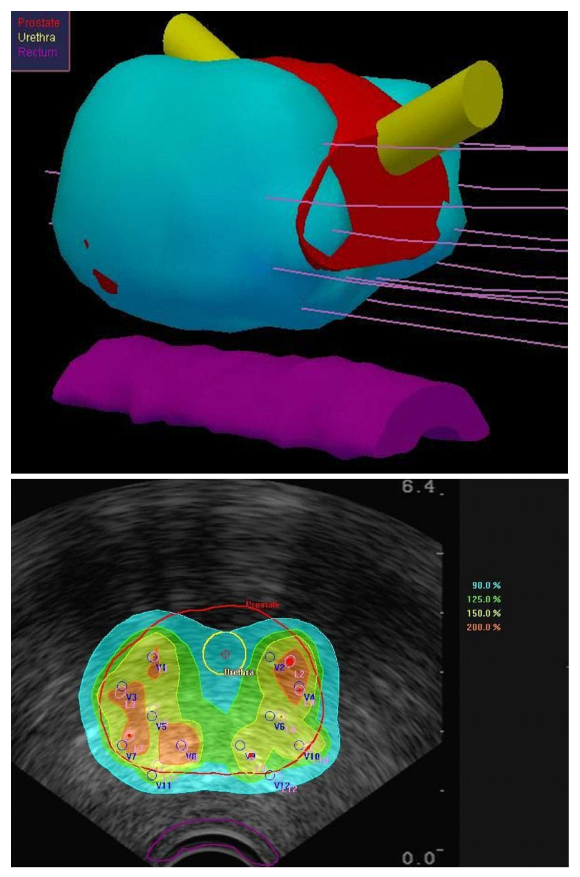

Fig. 2. (top) A three-dimensional representation of the live plan showing the calculated dose distribution (blue - $90 \%$ isodose) around the needles within which the source is moved, stopping at pre-selected dwell positions over optimised dwell times. The target volume, mostly "covered" by the $90 \%$ isodose is the prostate (red) and the critical organs are the rectum (violet) and urethra (yellow). (bottom) The central cross-sectional rectal ultrasonic image of the prostate also showing the needle positions (V1-V12) and the contours of the prostate (red), the urethra (yellow) and of the rectum wall (violet). Isodose distributions shown are $90 \%$ (blue), $125 \%$ (green), 150\% (yellow) and over 200\% (orange) of the prescribed dose. 


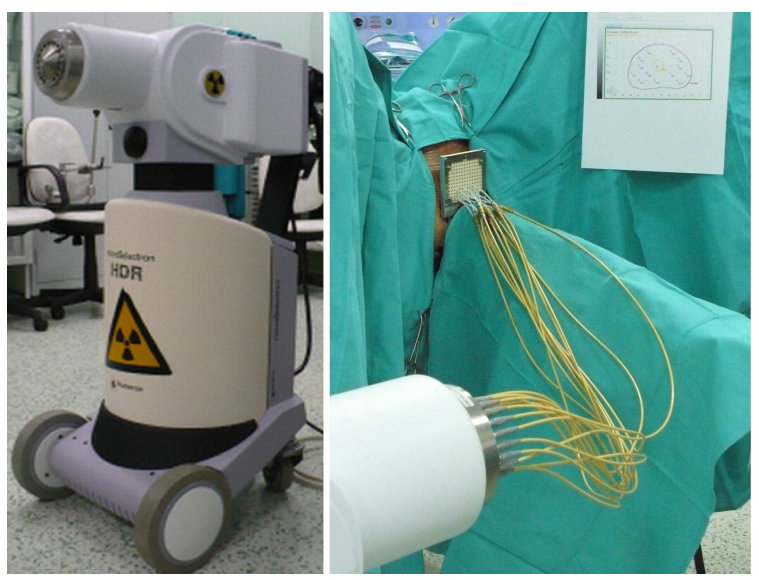

Fig. 3. (left) The MicroSelectron HDR afterloading unit - general view. (right) Shown are catheters joining the channel sockets in the 18-channel treatment head of the MicroSelectron HDR afterloading unit with the needles inserted in the patient which permit the Ir-192 source to be moved into successive needles by inserting or withdrawing the thin cable to which the source is attached. Source dwell positions are selected by suitable and accurate mechanical extension of the length of this cable, and dwell times by the source remaining in its given dwell position over the required period. Operation of this unit is fully controlled by data supplied by the live treatment plan accepted for treatment.

afterloading HDR therapy device (Fig. 3) which controls the source motion, its dwell positions and dwell times in order to realise the final (live) treatment plan of dose delivery to the patient. The HDR unit begins operation only after the medical staff leaves the HDR bunker.

The recommended total dose to the periphery of the target volume is 45 Gy for Ir-192, delivered in three 15 Gy sessions, repeated every three weeks, if HDR brachytherapy is the sole modality of radiation treatment. Each session involves the above-described procedure which lasts about $4 \mathrm{~h}$.

The equipment and software used to perform real-time HDR brachytherapy at our Centre of Oncology has been manufactured by Nucletron company (which calls it the SWIFT@-treatment). The treatment planning system is the Oncentra Prostate v. 3.0 and the irradiation device is the MicroSelectron HDR, an 18-channel remote afterloading unit with a single Ir-192 source of typical activity 10-20 Ci (Fig. 3). All equipment is interconnected via a dedicated network and all relevant data, such as patient data, ultrasound images, virtual and live treatment plans and treatment data, are recorded in a database system.

A detailed quality assurance programme has been implemented at COOK to guarantee the safety and repeatability of the performance of this equipment.

\section{Conclusions}

The general principle of applying brachytherapy alone or in combination with external beam treatment has been well established in gynaecological and head and neck cancers. With regard to delivery of a high radiation dose within a well-defined volume, brachytherapy may often be superior to sophisticated conformal or IMRT external radiotherapy techniques. The prostate is a good candidate for brachytherapy, especially if the described HDR technique is considered, however only in cases where the tumour is well localised and relatively small. In the treatment of early prostate cancer short-lived radionuclide sources (iodine-125 and palladium-103 seeds), emitting low energy photons $(\approx 30 \mathrm{keV})$ are often placed permanently around the tumour volume. The number of seeds required and their geometric placement in the target volume is determined through optimised computer dose planning or precalculated monograms [5]. The combination of brachytherapy and hyperthermia may in some cases lead to an improvement in the result of treatment $[6,7]$. At COOK we will introduce this treatment modality in the near future.

In a typical radiotherapy department some $10-20 \%$ of all radiotherapy patients are treated using various forms of brachytherapy. This modality allows excellent conformal treatment to be achieved without the complex accelerator and multileaf collimator technology and the need to apply highly advanced external therapy planning routines.

Treatment planning and patient treatment, based on ultrasound examinations, and HDR dose delivery is a new challenge to the medical physicist who now enters directly into the operating room and is expected to closely collaborate with the physician under stringent time limits. Good understanding of all aspects of this invasive treatment and the ability of the medical physicist to perform under considerable pressure are therefore essential.

\section{References}

[1] N. Suntharalingam, E.B. Podgorska, H. Tölli, Brachytherapy: Physical and Clinical Aspects, International Atomic Energy Agency, Vienna 2005.

[2] A. Śladowska, Acta Phys. Pol. A 115, 586 (2009).

[3] R. Makarewicz, Brachyterapy HDR, Via Media, Gdańsk 2004 (in Polish).

[4] K. Ślosarek, J. Bystrzycka, M. Fijałkowski, Rep. Pract. Oncol. Radiother. 10, 255 (2005).

[5] C. Salembier, P. Lavagnini, P. Nickers, P. Mangili, A. Rijnders, A. Polo, J. Venselaar, P. Hoskin, Radiother. Oncol. 83, 3 (2007).

[6] S. Maluta, S. Dall'oglio, M. Romano, N. Marciai, F. Pioli, M.G. Giri, P.L. Benecchi, L. Komunale, A.B. Porcaro, Int. J. Hyperthermia 23, 451 (2007).

[7] N. Piotrkowicz, J. Zieliński, P. Dębicki, J. Jońska, Rep. Pract. Oncol. Radiother. 10, 131 (2005). 